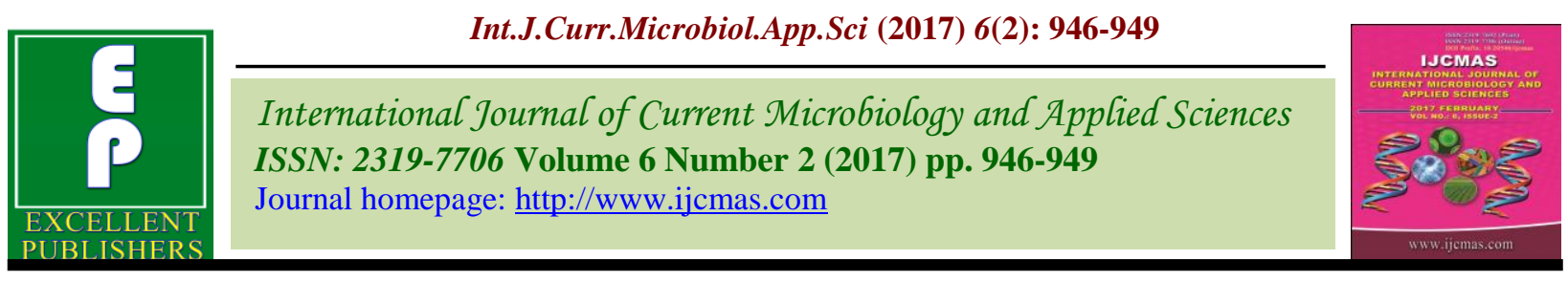

Case Study

http://dx.doi.org/10.20546/ijcmas.2017.602.105

\title{
Isolation of Sporothrix schenckeii from Ear Discharge - An Unusual Presentation of Sporotrichosis
}

\section{Purbasha Ghosh $^{1 *}$, Paulami Ghosh ${ }^{2}$, Suvodip Chakraborty ${ }^{2}$, Asraful Islam ${ }^{2}$, Indrait Gupta ${ }^{2}$ and Satrajit Roy ${ }^{2}$}

${ }^{1}$ Department of Microbiology, Burdwan Medical College, WB, India

${ }^{2}$ Department of Microbiology, Burdwan Medical College, WB, India

*Corresponding author

\section{A B S T R A C T}

\section{Keywords \\ Sporotrichosis, \\ CSOM, \\ Occupational \\ disease. \\ Article Info \\ Accepted: \\ 20 January 2017 \\ Available Online: \\ 10 February 2017}

\section{Introduction}

Sporotrichosis is chronic fungal infection follows the implantation of spores in a penetrating wound (Bhutia et al., 2011). Sporothrix schenckeii grows in soil or vegetations (Rei et al., 2015). It is regarded as occupational disease (Lederer et al., 2016). But here we report a case of mucocutaneous sporotrichosis of ear in a 30yrs old housewife leads to chronic suppurative otitis media (CSOM).

\section{Case report}

A 30 years old housewife from a village of Burdwan District came ENT OPD with complain of suppurative ear discharge from left ear for more than 3 weeks along with dull ear ache and on and off fever. Otoscopic findings revealed chronic suppurative otitis media (CSOM) and the swabbing of the seropurulent ear discharge was sent to the Microbiology Laboratory for further investigations. Direct staining of the sample was followed by the inoculation of the sample on both bacterial and fungal culture media. Gram's stain showed Gram positive cocci in clusters with few pus cells. $\mathrm{KOH}$ mount showed some elongated yeast cells. Colonies of Staphylococcus aureus was appeared on Blood agar. SDA (Sabouraud's Dextrose Agar) culture at $25^{\circ} \mathrm{C}$ showed black colonies within a week (Picure 1). Lactophenol cotton 
blue (LPCB) gave the typical 'Twisted rope' appearance of septate hyphe with flower like sporulation [Picture 2(i) and 2(ii)]. SDA culture at $37^{\circ} \mathrm{C}$ showed the cream coloured colonies (Picture 3) Gram's stain of that colonies showed ellipsoidal yeast cells (Pic4). The demonstration of dimorphism and other associated features proved the identification of Sporothrix schenckeii.

Staphylococcus aureus was also isolated causing CSOM. Subsequently the patient was responded well with the therapy of saturated solution of potassium iodide (SSKI) along with antibiotic coverage.

\section{Results and Discussion}

We hypothesize that, the source of infection was straw from field as the patient had a habit of using straw stick for removing ear-wax. The mode of entry can be through scratches or blunt injury. It has been known that a humid and moist climate favours the growth of S. schenckii (Motswaledi et al., 2011). The ear cavity gave the perfect enrichment for Sporothrix. The infection was typically chronic and may favour the super infection of CSOM by Staphylococcus aureus. It usually occurs in people occasionally exposed to outdoor works like mine workers, farm workers, gardeners and florists (Xavier et al., 2013).

Pic.1 showing the black colonies on SDA at $25^{\circ} \mathrm{C}$

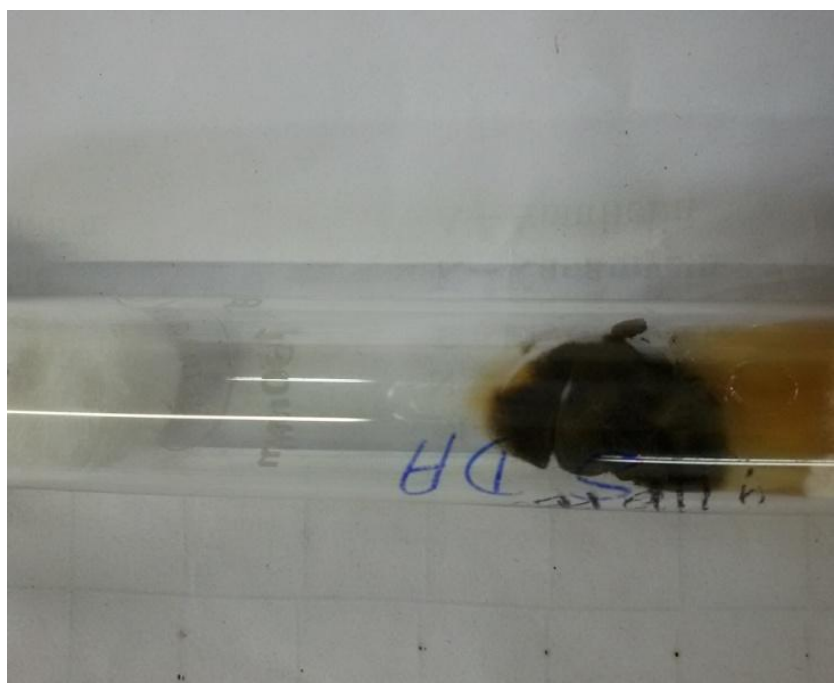

Pic.2a LPCB stain shows 'Twisted rope' appearance (10x)

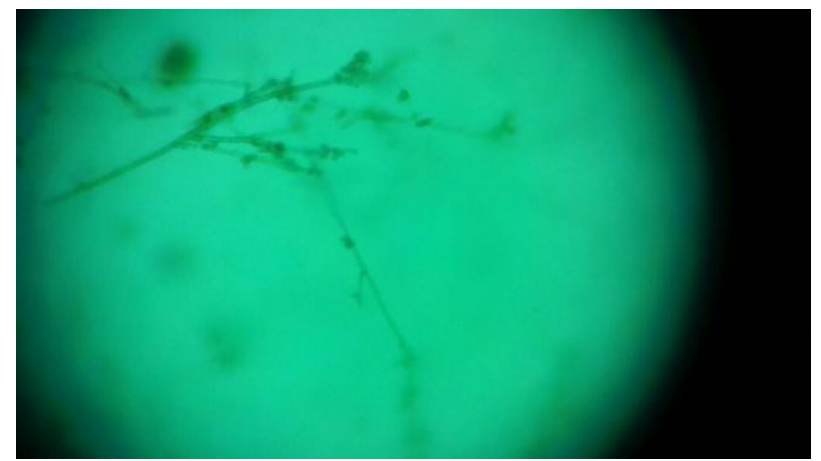


Pic.2b LPCB stain shows flower like appearance (40x)

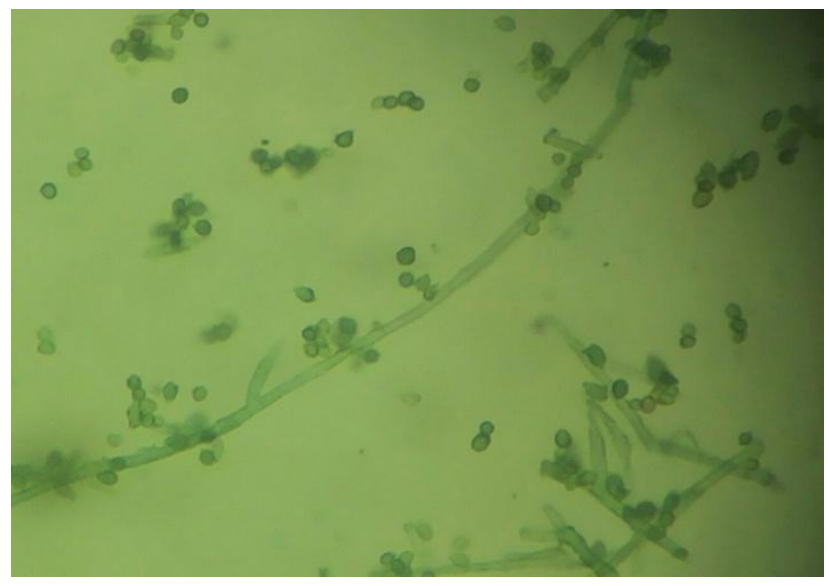

Pic.3 Cream coloured colonies on SDA at $37{ }^{\circ} \mathrm{C}$

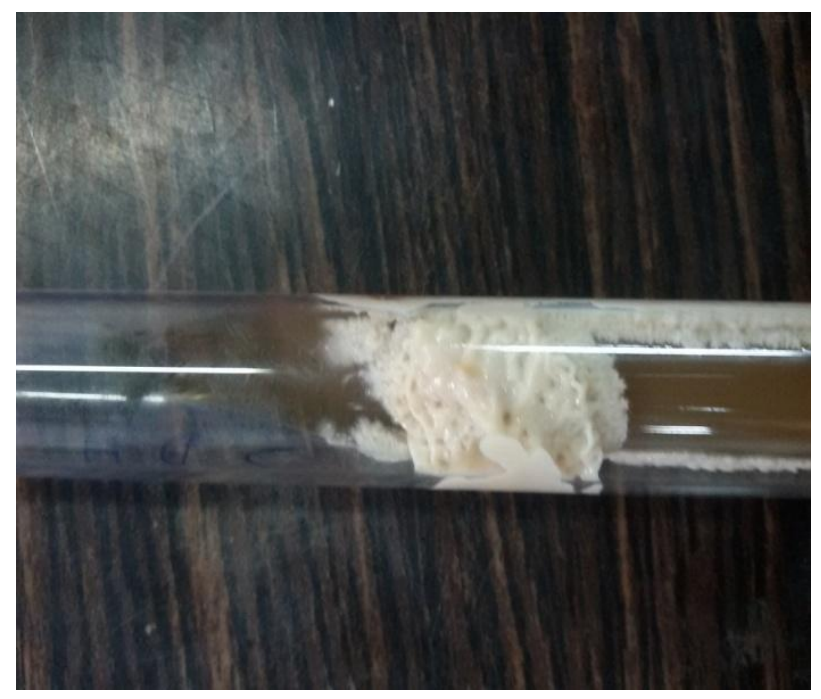

Pic.4 Gram's stain shows yeast cells

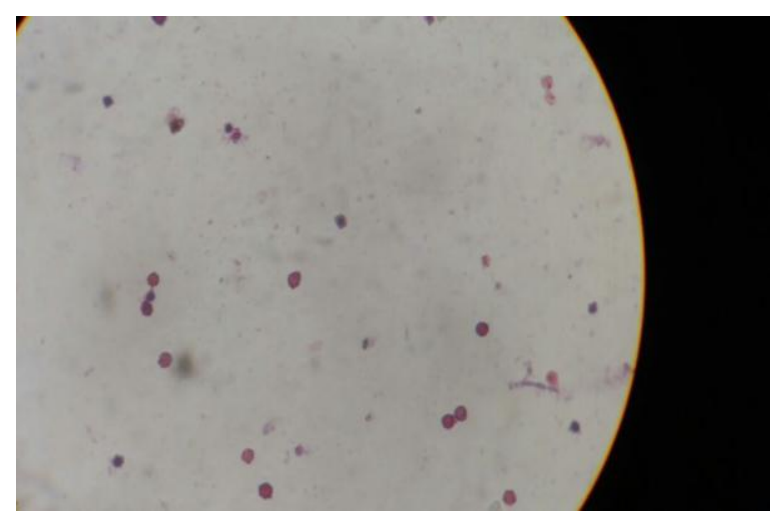


But here the patient is housewife. Mucocutaneous type of sporotrichosis is occasional and generally occurs on mouth, pharynx and nose. Pain is common in mucoctaneous type which is rare in cutaneous type. We must remark that the gold standard for diagnosis of sporotrichosis is mycological culture for fungi isolation and identification with relevant clinical presentation (Wang et al., 2000). Melanization also has a role in the pathogenesis of cutaneous sporotrichosis, (Monica et al., 2011). Since pigmented isolates had a greater invasive ability (Coskun et al., 2004).

In conclusion, we describe a case of mucocutaneous sporotrichosis in an uncommon location with an unusual mode of transmission. The case report describes the risk factor, diagnosis, and treatment of this unusual infection. Sporotrichosis should be considered in the differential diagnosis of such clinical presentation. The aim is early diagnosis and treatment. Awareness of this disease and an extensive environmental study is required to understand the epidemiology. Here we emphasize the disease importance. To the best of our knowledge, this is the first reported case from West-Bengal.

\section{References}

Bhutia, P.Y., Gurung, S., Yegneswaran, P.P., Pradhan, J., Pradhan, U., Peggy, T., Pradhan, P.K., Bhutia, C.D. 2011. A case series and review of sporotrichosis in Sikkim. J. Infect. Dev. Ctries, 5(8): 603-608.
Coskun, B., Saral, Y., Akpolat, N., Ataseven, A. \& Demet, C. Sporotrichosis successfully treated with terbinafine and potassium iodide: Case report and review of the literature. Mycopathologia, 158: 53-56.

Lederer, H.T., Sullivan, E., Crum-Cianflone, N.F. 2016. Sporotrichosis as an unusual case of osteomyelitis: A case report and review of the literature. Med. Mycol. Case Report, 11: 31-35.

Monica Bastos de Lima Barros, Rodrigo de Almeida Paes and Armando Oliveira Schubach. 2011. Sporothrix schenckii and Sporotrichosis. Clin. Microbiol. Rev., 24(4): 633-654.

Motswaledi, H., Nkosi, L., Moloabi, C., Ngobeni, K., Nemutavhanani, Maloba. 2011. Sporotrichosis: A Case Report and Literature Review. Clin. Experimental Dermatol. Res., 2: 7.

Rei, B.D., Cobucci, F.O., Zacaron, L.H., D’Acri, A.M., Lima, R.B., Martins, C.J. 2015. Sporotrichosis in an unusual location - Case report. An. Bras. Dermatol., 90(3 Suppl 1): S84-7.

Wang, J.P., Granlund, K.F., Bozzette, S.A., Botte, M.J., and Fierer, J. Bursal Sporotrichosis: Case Report and Review. Clin. Infect. Dis., 31: 615-6.

Xavier, M.O., Bittencourt, L.R., Silva, C.M., Vieira, R.S. and Pacheco Pereira, H.C. 2013. Atypical presentation of sporotrichosis: report of three cases. Revista da Sociedade Brasileira de Med. Trop., 46(1): 116-118.

\section{How to cite this article:}

Purbasha Ghosh, Paulami Ghosh, Suvodip Chakraborty, Asraful Islam, Indrait Gupta and Satrajit Roy. 2017. Isolation of Sporothrix schenckeii from Ear Discharge - An Unusual Presentation of Sporotrichosis. Int.J.Curr.Microbiol.App.Sci. 6(2): 946-949. doi: http://dx.doi.org/10.20546/ijcmas.2017.602.105 